\title{
The Search for Air Overpressure During Blasting Operations
}

\section{Phil Nesbitt, LBNF/DUNE - US, Mining Engineer Intern}

\section{Underground Blasting Criteria}

While utilizing explosives underground has many safety considerations, one of the unique limits imposed on blasting at the LBNF/DUNE-US site involves keeping the air pressure/noise from blasting operations below $133 \mathrm{~dB}$ in the vicinity of the CASPAR laboratory. This is to ensure the safety and integrity of sensitive equipment inside.

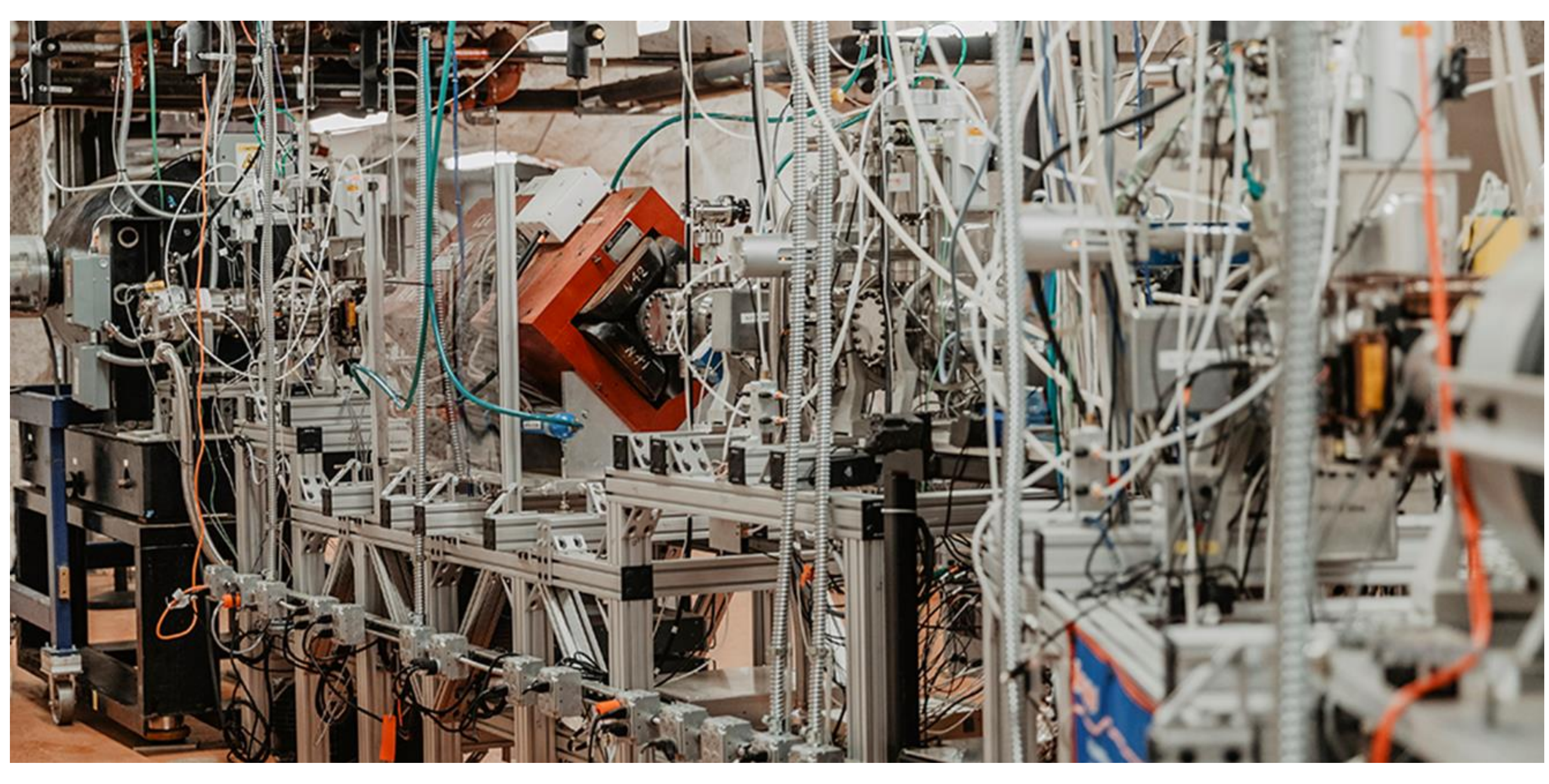

CASPAR - Photo by Nick Hubbard

During blasting of the machine shop egress (4850-90A) which is located right next to sensor 3 , air overpressure readings were recorded on sensor 6 which is located directly outside of CASPAR. These readings were not duplicated on any other sensor causing some confusion. Several sensors are positioned such that the soundwave would pass them before arriving at sensor 6 meaning energy should dissipate by arrival at sensor 6 , not increase. Another consideration is the location of blast doors in relation to the various sensors.

\section{Fermilab}

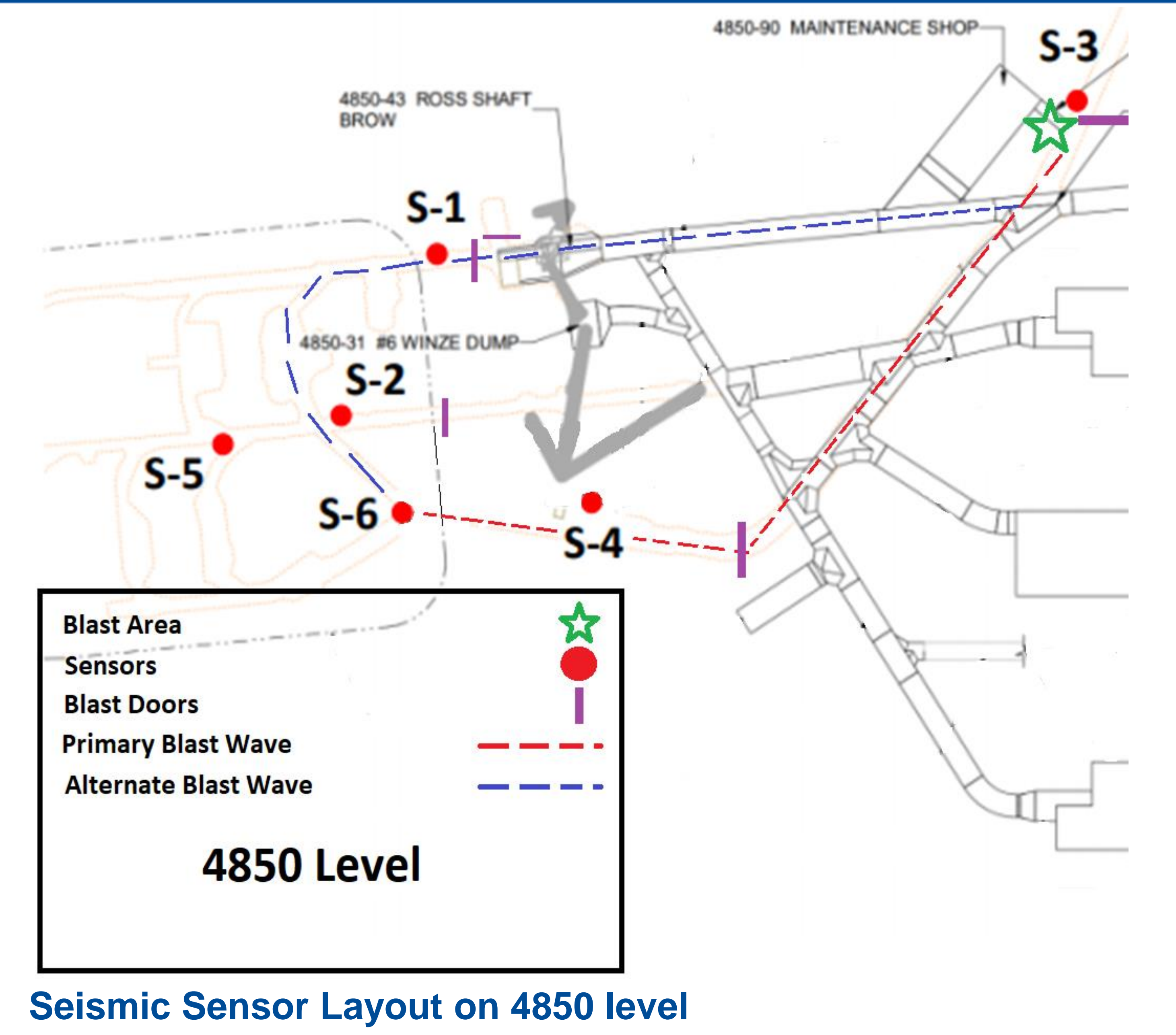

Analyzing the data, the overpressure acoustic range was between 1.5 and $7.2 \mathrm{~Hz}$ with most overpressures falling near the $3.5 \mathrm{~Hz}$ range. The average time the overpressure was recorded was 3.23 seconds.

Acoustically speaking, a soundwave travelling in air from the blast, taking the most direct route, should arrive at sensor 6 within 1.9 seconds. That this time was almost doubled was a clue that multi-path sound propagation was occurring. Also, the possibility that different waveforms were reinforcing each other was tantalizing.
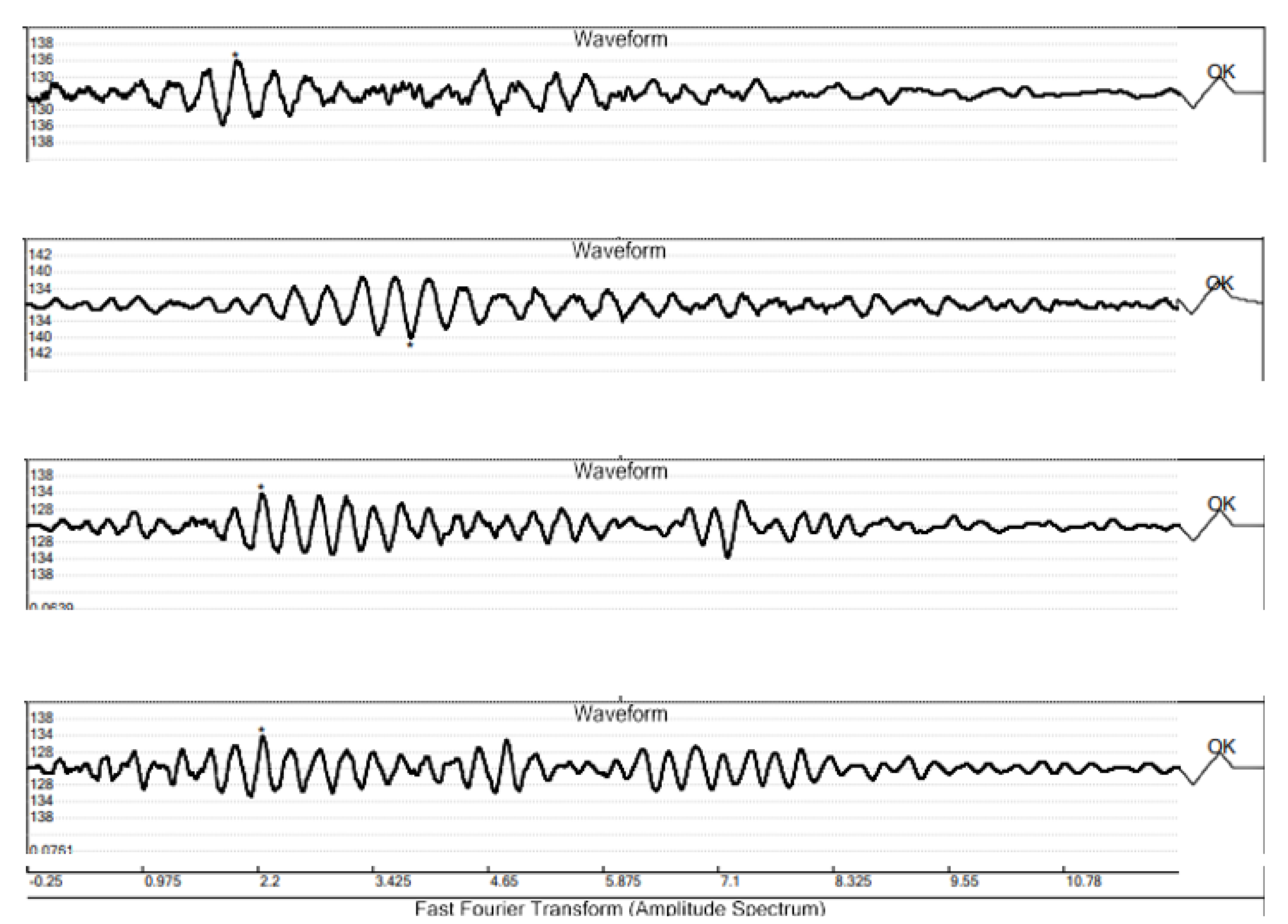

Acoustic Comparison for over-pressure readings on Sensor 6

\section{FERMILAB-POSTER-21-030-LBNF}

Another data point analyzed was the waveforms appeared sinusoidal. This is an indication that a resonance was present. In taking both the resonance and the multipath sound propagation as primary data points, an experiment was conducted where-by a video was recorded of airflow indicators which were installed in the vicinity of sensor 6 to determine the direction and intensity of the airflow after the blast. The next step was moving the sensor from the metal post it had been mounted to and attach it to the concrete wall for measurement during a subsequent blast.

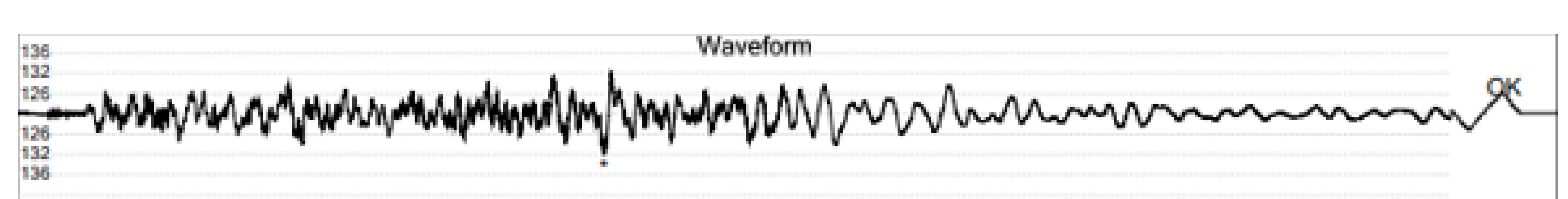

Post-Corrective action Acoustic reading demonstrating lack of sinusoidal wave.

\section{Conclusion}

Once the sensor was removed from the metal post, the sinusoidal nature of the soundwaves disappeared indicating that the post was likely resonating with the frequency of the soundwave causing a false overpressure reading. The airflow video showed a large volume of air was moving rapidly through the area indicating that air travel was likely flowing through several adjacent levels and returning through the \#6 winze during the blast. This helped to explain why the sound took longer than expected to arrive at the sensor. It was determined that resonance was the most likely culprit. The microphone was replaced and remains mounted to the concrete wall. In all subsequent blasts, no overpressure has been recorded that was not attributed to the type and weight of explosives being used.

Acknowledgements: Joshua Willhite - Design Manager; James Rickard - EXC Resident Engineer; Aaron Schwan - Intern 